\title{
Apreçando Derivativos de Crédito no Brasil
}

\author{
Jorge C. Kapotas* \\ Pedro Paulo Schirmer** \\ Marcelo M. Taddeo****
}

Resumo

Neste artigo apresentamos os principais modelos de apreçamento de instrumentos de crédito onde desenvolvem-se as equações de apreçamento de debêntures. Para tal, utilizam-se os modelos estruturais, de intensidade e, finalmente, de rating. A seguir, definimos o que são derivativos de crédito (CDS) e derivamos fórmulas de apreçamento para estes instrumentos. Concluímos este artigo com uma aplicação ao mercado brasileiro.

\begin{abstract}
In this paper we present the main models used for pricing defaultable bonds and credit derivatives. The Merton structural model, the intensity framework and a Ratings based model are considered. We apply these techniques to the pricing of credit derivatives on brazilian US\$-indexed treasury bonds.
\end{abstract}

Palavras-chave: derivativos de crédito; debêntures; swaps de crédito, spreads.

Códigos JEL: G12; G13.

\section{Introdução}

Nos mercados internacionais, os derivativos de crédito iniciaram seu desenvolvimento, enquanto mercado e produto, a partir dos tradicionais asset swaps dos anos 90. Já em 1995 registraram-se os primeiros credit default swaps e em 1997 os derivativos de crédito detinham um mercado de USD 187 bilhões correspondentes ao total de contratos em aberto naquele ano. Em junho de 2004 a ISDA $^{1}$ registrou um total de USD 5,44 trilhões para o valor total de contratos em aberto até aquela data. Esta vertiginosa taxa de crescimento de volumes está solidamente ancorada na alta de liquidez dos mercados internacionais de títulos de renda fixa com risco de crédito, os quais, por sua vez, permitem a formação de carteiras replicantes. ${ }^{2}$

Em função do imponente crescimento dos mercados de derivativos de crédito, inúmeros trabalhos e artigos dedicados à análise e apreçamento destes instrumentos foram produzidos no meio acadêmico. Os trabalhos Duffie e Singleton (1999), Schonbucher (2000), Hull e White (2000) e Belangier et alii (2001) são exemplos

\footnotetext{
Submetido em Julho de 2004. Revisado em Novembro de 2004.

*Octaplus Financial Analytics. Rua Quintana, 753, $9^{\circ}$ andar. CEP: 04569-011, São Paulo, Brasil

**Instituto de Matemática e Estatística, Universidade de São Paulo. Rua do Matão, 1010 CEP: 05508-090, São Paulo, Brasil.

***Instituto de Matemática e Estatística, Universidade de São Paulo. Rua do Matão, 1010. CEP: 05508-090, São Paulo, Brasil.

${ }^{1}$ International Swaps and Derivatives Association, Inc.

${ }^{2}$ Para as relações de arbitragem impostas pelas carteiras replicantes dos swaps de crédito e suas implicações em seu apreçamento, ver Duffie (1996) e hedges para as posições dos market-makers.
} 
típicos dos trabalhos publicados nesta direção. Recentemente, tem-se observado no Brasil um crescente interesse por parte dos agentes do mercado financeiro nos derivativos de crédito. De fato, os swaps de crédito (credit default swaps) já estão devidamente regulamentados pelas autoridades competentes ${ }^{3}$ e sua liquidação será feita pela CETIP. ${ }^{4}$

No entanto, o desenvolvimento dos mercados brasileiros de derivativos de crédito vem sendo penalizado pela reduzida liquidez dos instrumentos de crédito no Brasil. Por outro lado vale a pena salientar que no ano de 2004 registrou-se um significativo aumento no volume de emissões de debêntures, sendo estimado até o final do mesmo um total acumulado de R $\$ 15$ bilhões de emissões no mercado primário de debêntures, ou seja três vezes superior ao volume de $2003 .{ }^{5}$ Tal fenômeno reflete a crescente demanda por parte de investidores institucionais e privados por ativos negociáveis de crédito, capazes de proporcionar taxas esperadas de retorno mais atrativas em uma conjuntura de taxas de juros declinantes.

Neste artigo são apresentados os principais modelos de apreçamento de instrumentos de crédito, onde desenvolvem-se as equações de preço de debêntures (seção 2). Para tal, são utilizados modelos estruturais, de intensidade e, finalmente, de rating. A seguir, definimos um derivativo de crédito, CDS, (seção 3) e derivamos sua fórmula de apreçamento (seção 4). Concluímos este artigo com uma aplicação para o mercado brasileiro (seção 5).

\section{Modelos de Apreçamento de Instrumentos de Crédito}

Nosso objetivo nessa seção é apresentar de forma rápida e sucinta as principais classes de modelos de apreçamento de títulos com risco de crédito desenvolvidos até o momento. Pode-se dividir de forma simplificada tais modelos em três categorias:

- modelos estruturais ou da firma;

- modelos de intensidade;

- modelos de rating.

Os modelos estruturais têm sua origem no artigo Merton (1974). Estes modelos utilizam o arcabouço teórico de apreçamento de opções elaborado originalmente em Black e Scholes (1973). No seu trabalho seminal Merton considera uma firma teórica cujo passivo é composto por um único título negociável de renda fixa e uma ação, ambos apreçados pelo mercado. Várias e importantes condições são impostas ao modelo, como veremos abaixo, no sentido de adequar o formalismo

\footnotetext{
${ }^{3}$ Ver a resolução 2933/02 do CMN que autoriza a operaçõ de derivativos de crédito e a circular 3106/02 do BACEN que dispõe sobre a realização das operações de derivativos de crédito que trata a Resolução 2933/02.

${ }^{4}$ Central de Custódia e de Liquidação Financeira de Títulos Privados. As informações operacionais sobre os procedimentos de liquidação podem ser obtidos em: www.cetip.com.br

${ }^{5}$ Ver Jornal Valor, caderno de Finanças, 2/08/2004.
} 
em tempo contínuo de Black-Scholes do apreçamento de opções ao problema do apreçamento de títulos negociáveis de crédito. Nesta classe de modelos o valor total dos ativos de uma firma é usado para determinar o evento de default, que ocorre quando o valor dos ativos decresce até um nível crítico de default. Os modelos estruturais são, portanto, de natureza endógena, como será visto na subseção 2.1 .

Nos modelos de intensidade, a dinâmica do valor dos ativos da firma não é modelada diretamente, sendo o default determinado por um processo pontual caracterizado por uma taxa de sinistro ou intensidade de default. Este tipo de abordagem ao problema de crédito encontra representação nos clássicos trabalhos Jarrow e Turnbull (1995), Lando (1998) e Duffie (1999), entre vários outros, onde o default é modelado de maneira exógena. Estes modelos são tratados em maior profundidade na subseção 2.2. Com o objetivo de ilustrar a aplicação destes modelos no apreçamento de derivativos de crédito, utilizamos uma versão particular de um modelo de intensidade para o apreçamento analítico de um swap de crédito na seção 4, e sua posterior aplicação concreta no mercado brasileiro de títulos cambiais feita na seção 5 deste artigo.

Os modelos de rating, também conhecidos por modelos Markovianos de migração de crédito, objetivam por sua vez modelar a dinâmica das transições de rating no tempo de um título de crédito dentre os vários ratings possíveis de serem atingidos. Para tanto as transições de rating são modeladas em termos de uma cadeia Markoviana com espaço de estados finito e o estado correspondente ao evento de default torna-se neste caso absorvente, evitando-se assim múltiplos defaults. Estes modelos têm como peça fundamental a matriz de probabilidades de transição de ratings como veremos na subseção 2.3. O trabalho de Jarrow et alii (1997) representa bem esta classe de modelos.

\subsection{Modelos estruturais}

Como já foi observado, os modelos estruturais seguem o modelo básico de Merton $^{6}$ e assumem as seguintes premissas (algumas das quais podendo ser enfraquecidas, como será visto na subseção 2.1.1):

- o mercado é perfeito, livre de arbitragem e sem atrito, ou seja, não há custos de transação;

- taxa livre-de-risco conhecida e constante;

- os ativos da firma são perfeitamente divisíveis e liquidamente negociáveis em mercado;

- o valor dos ativos da empresa no instante $t, V_{t}$, segue um processo difusivo com dinâmica log-normal:

${ }^{6}$ Ver desenvolvimento original em Merton (1974), e em Bielecki e Rutkowski (2002) e Cossin e Pirotte (2000) entre os mais recentes. 


$$
\frac{d V_{t}}{V_{t}}=\mu d t+\sigma_{V} d W_{t}
$$

onde $\mu$ é a taxa de retorno instântanea do ativo, $\sigma_{V}$ sua volatilidade e $W_{t}$ representa o processo de Wiener unidimensional ao longo do tempo $t$;

- as hipóteses do teorema de Modigliani-Miller (MM) são consideradas válidas, ou seja, os fluxos de caixa da empresa satisfazem a equação

$$
V_{t}=D_{t}+S_{t}
$$

onde $D_{t}$ representa a dívida vencendo na data terminal $T$ e $S_{t}$ o valor das ações da empresa;

- os investidores são racionais, ou seja, desejam maximizar sua riqueza;

- a empresa não se encontra em default no instante inicial $t$ e não existem pagamentos intermediários antes de $T$ aos investidores da empresa. Em outras palavras, o default só pode ocorrer no vencimento $T$ e os acionistas recebem nesse momento o valor residual da empresa após a liquidação da dívida. Mais ainda, não há custos de falência.

O próximo passo será a introdução do conceito de opções no modelo da firma. Suponha que a empresa emite uma única debênture cujo valor de face é $F$. Denotando por $D_{s}$ seu valor num instante $s>t$, é fácil ver que:

- se $V_{T} \leq F$ :

o titular da debênture deve receber $V_{T}$ e os acionistas da empresa não devem receber nada. Ou seja,

$$
\begin{aligned}
D_{T} & =V_{T} \\
S_{T} & =0
\end{aligned}
$$

e o valor da perda por parte dos debenturistas será $F-V_{T}$. Consequentemente, pode-se pensar a perda dos debenturistas, neste caso, é dada por

$$
\max \left(F-V_{T}, 0\right)
$$

que é igual ao valor do payoff de uma opção de venda; 
- se $V_{T}>F$ :

nesse caso o debenturista deve receber $F$, enquanto que os acionistas deverão ficar com $V_{T}-F$, ou seja,

$$
\begin{aligned}
D_{T} & =F \\
S_{T} & =V_{T}-F
\end{aligned}
$$

de modo que o valor recebido pelos acionistas é dado por

$$
\max \left(V_{T}-F, 0\right)
$$

que é igual ao payoff de uma opção de compra.

Segue da equação (4) que

$$
S_{t}=C\left(V_{t}, F, r, T-t, \sigma_{V}\right)
$$

e da equação (3) que a perda dos debenturistas é dada por

$$
P\left(V_{t}, F, r, T-t, \sigma_{V}\right)
$$

onde $C\left(V_{t}, F, r, T-t, \sigma_{V}\right)$ é a fórmula de Black-Scholes para preços de opções de compra, e $P\left(V_{t}, F, r, T-t, \sigma_{V}\right)$ é a fórmula para preços de opções de venda. Naturalmente, $V_{t}$ exerce o papel do ativo base da opção, enquanto que $F$ está no lugar do preço de exercício. Inserindo a expressão (5) na fórmula (2) de ModiglianiMiller segue que

$$
D_{t}=V_{t}\left[1-N\left(d_{1}\right)\right]+F e^{-r(T-t)} N\left(d_{2}\right)
$$

onde $N(\cdot)$ é a função distribuição de probabilidade normal padronizada. Os parâmetros $d_{1}$ e $d_{2}$ são, evidentemente, os mesmos da equação de apreçamento de Black-Scholes. ${ }^{7}$ A expressão (7) é denominada fórmula de Merton para o valor de uma debênture com risco de crédito. Manipulando-se algebricamente a fórmula de Merton, obtém-se

$$
D_{t}=F_{e}^{-r(T-t)}-\underbrace{\{N\left(-d_{2}\right) \underbrace{\left[F_{e}^{-r(T-t)}-V_{t} \frac{N\left(-d_{1}\right)}{N\left(d_{2}\right)}\right]}\}}_{\text {Perda dado default }}
$$

Perda esperada

\footnotetext{
${ }^{7}$ Ver Black e Scholes (1973)
} 
Vemos assim que a fórmula (8) evidencia o caráter endógeno do modelo, capaz de fornecer intrinsicamente não somente a perda esperada como também a taxa de recuperação implícita.

Nos mercados de renda fixa é natural trabalharmos com yields. Assim, considere a taxa (yield) $y$, tal que $D_{t}=F e^{-y(T-t)}$, e defina o spread de crédito por

$$
\xi_{t}=y_{t}-r_{t}
$$

Então, substituindo $y_{t}$ por $-\frac{\log \left(\frac{D_{t}}{F}\right)}{T-t}$ em (9) e usando a expressão resultante da fórmula de Merton (7) tem-se a seguinte expressão para o spread de crédito:

$$
\xi_{t}(T)=\frac{-1}{T-t} \log \left[N\left(d_{2}\right)+\frac{V_{t}}{F e^{-r(T-t)}} N\left(-d_{1}\right)\right]
$$

Como visto acima, considerando-se o valor das ações da empresa como uma função dos seus ativos, $S=S(V)$, obtemos uma expressão para a volatilidade dos ativos da empresa em função da volatilidade de suas ações:

$$
\sigma_{V}=\frac{S_{t}}{V_{t}}\left(\frac{1}{\frac{\partial S}{\partial V}}\right) \sigma_{S}
$$

A fórmula acima é o resultado da aplicação da fórmula de Itô em $S(V)$ considerando que $V_{t}$ satisfaz a equação diferencial estocástica (1).

\subsubsection{Enfraquecendo hipóteses}

O modelo de Merton assume um grande número de hipóteses, algumas excessivamente restritivas, causando uma significativa redução na sua aplicabilidade e no poder preditivo do modelo. Com o intuito de se reduzir essas limitações foram elaboradas extensões do modelo básico de Merton enfraquecendo-se algumas hipóteses ou premissas do mesmo. ${ }^{8}$ Entre eles destacamos os seguintes modelos:

- Black e Cox (1976);

- Leland (1994);

- Brys e De Varenne (1997).

No modelo de Black-Cox assume-se que:

\footnotetext{
${ }^{8}$ Ver especialmente Black e Cox (1976) e Vasicek (1984); Brys e De Varenne (1997) e Shimko et alii (1993) para taxas de juros estocásticas e Zhou (1996) para uma abordagem utilizando um processo de difusão com saltos para a dinâmica do valor da firma.
} 
- o evento de crédito pode ocorrer antes do vencimento $T$ e neste modelo significa que o valor dos ativos da firma atingiu um determinado valor crítico. O tempo de default será portanto modelado por uma variável aleatória $\tau$, que fornece o primeiro instante de ocorrência deste evento;

- o valor crítico de default dos ativos da firma $\nu(\cdot)$ não necessita ser constante no tempo, podendo ser uma função determinística do tempo da forma $\nu(t):=K e^{-\gamma(T-t)}$ onde $K$ é um valor constante e $\gamma$ é um fator de crescimento;

- existem frações variáveis de recuperação $\beta_{1}$ e $\beta_{2}$ tal que $0 \leq \beta_{1}, \beta_{2} \leq 1$, as quais podem violar a premissa de Merton de ordenamento de pagamento dos passivos. Tais variáveis não priorizam o pagamento aos detentores de passivos da firma, embutindo na equação os custos de liquidação da empresa pagos a terceiros.

Nesse modelo o preço da debênture resulta da seguinte soma de valores esperados:

$$
\begin{aligned}
D(t, T) & =F e^{-r(T-t)} \mathbb{P}\left[\tau \geq T, V_{T} \geq F\right] \\
& +\beta_{1} V_{T} e^{-r(T-t)} \mathbb{P}\left[\tau \geq T, V_{T}<F\right] \\
& +\beta_{2} K e^{-\gamma(T-\tau)} e^{-r(\tau-t)} \mathbb{P}[t<\tau<T]
\end{aligned}
$$

A equação (12) representa o valor esperado dos três cenários possíveis para a debênture no modelo de Black-Cox: o primeiro termo representa o cenário de solvência da firma, o segundo termo representa o cenário de insolvência da firma no vencimento e o útimo reflete a possibilidade de default da firma antes do vencimento do título.

As probabilidades acima podem ser calculadas analiticamente e para o cálculo da terceira probabilidade é utilizada a distribuição do tempo de absorção pela barreira definida pelo valor crítico $\nu(t){ }^{9}$

No modelo de Leland (1994) assume-se que a firma emite uma debênture perpétua que paga um cupom $c$ enquanto a firma está solvente. O default ocorre quando o processo dos ativos $V_{t}$ atinge um valor mínimo fixo $\nu$. Além de considerar os efeitos dos custos de liquidação, o modelo permite mensurar os efeitos dos benefícios fiscais gerados pelos juros da dívida no valor da firma. Esta abordagem permite que se analise no contexto dos modelos estruturais mais amplos as questões de estrutura ótima de capital das empresas. ${ }^{10}$

Já o modelo de Brys e De Varenne (1997) generaliza o modelo de Black-Cox incorporando taxas de juros estocásticas. Em outras palavras, as taxas de juros

\footnotetext{
${ }^{9}$ Por uma questão de espaço referenciamos o leitor ao trabalho original Black e Cox (1976) onde se encontra a fórmula completa para o preço de uma debênture.

${ }^{10}$ Do modelo de Leland (1994) pode-se extrair o valor ótimo $E_{t}^{*}$ da ação da firma, bem como o valor ótimo $D_{t}^{*}$ da dívida da empresa.
} 
livre de risco obedecem a uma dinâmica determinada por uma equação diferencial estocástica. Mais precisamente o modelo de Briys e de Varenne usa o modelo de Hull-White de um fator ajustado a estrutura a termo em $t$, com volatilidade constante $\sigma_{t}=\sigma$.

\subsubsection{Aplicabilidade e performance dos modelos estruturais}

Embora os modelos acima sejam intuitivos e relativamente simples, os mesmos apresentam sérias dificuldades de implementação. Os ativos da firma não sendo perfeitamente divisíveis e negociáveis quebram a condição de apreçamento por não-arbitragem, de essencial importância ao modelo. $\mathrm{O}$ valor da firma $V_{t}$ depende de $D_{t}$, o qual queremos avaliar, e $\sigma_{V}$ que é um parâmetro importante para esta classe de modelos denota a volatilidade de uma variável não-observável, devendo ser estimada em um sistema de equações não-lineares a partir da volatilidade de mercado da ação da empresa. No mundo real existe uma grande variedade de classes de passivos de empresas, em frontal oposição à firma teórica de Merton, o que torna necessário a utilização de informações contábeis, nem sempre disponíveis e/ou confiáveis, e métodos ad-hoc de adequação da informação disponível aos parâmetros do modelo. Estas questões inerentes à esta classe de modelos traduzem-se em uma baixa aderência econométrica à realidade dos mercados. ${ }^{11}$ De fato, o apreçamento dos instrumentos de crédito é completamente endógeno e dos modelos estruturais resultam os spreads teóricos de crédito. A comparação destes aos spreads de mercado permite avaliar o poder preditivo desta classe de modelos.

No Brasil, a situação agrava-se em decorrência da complexa estrutura de passivos das empresas locais e do pequeno número de empresas de capital aberto, fazendo com que a aplicação de modelos estruturais torne-se ainda mais problemática.

\subsection{Modelos de intensidade}

Os modelos de intensidade, introduzidos por Jarrow e Turnbull (1995) e também por Duffie e Singleton (1999), entre outros, consistem em focar sua atenção no momento de default ou evento de crédito. O evento de crédito é o momento em que se torna público ao mercado a incapacidade de uma empresa honrar suas dívidas. Desse modo, considera-se o tempo de default como sendo uma variável aleatória positiva e estuda-se sua distribuição de probabilidade. Por essa razão, modelos de intensidade também podem ser chamados de modelos de default.

Para definirmos formalmente um modelo de intensidade, consideramos um espaço de probabilidade filtrado $\left(\Omega, \mathbb{F}=\left\{\mathbb{F}_{t}\right\}, \mathbb{Q}\right)$ grande o suficiente para abrigar as possíveis realizações das variáveis macroeconômicas do modelo como, por exemplo, as taxas de juros, e as realizações de um processo de contagem $\left\{N_{t}\right\}_{t \geq 0}$ modelado por uma distribuição exógena.

\footnotetext{
${ }^{11}$ Veja em Ericsson e Reneby (2001), Ericsson e Reneby (1998), Jones et alii (1984), Lardic e Rouzeau (1999) e Bohn (1999) os resultados de testes empirícos dos modelos estruturais
} 
Para modelar o tempo de default com um processo pontual ou de contagem, usualmente faz-se uso de dos seguintes processos: ${ }^{12}$

- processo de Poisson $-\lambda$ (constante);

- processo de Poisson não-homogêneo - $\lambda(t)$ (determinístico);

- processo de Cox $-\lambda_{t}$ (estocástico).

Dada a natureza da variável aleatória $\tau$ é natural que se use um processo de Poisson para modelá-la. Mais ainda, a modelagem do tempo de default por meio de um processo de Poisson não-homogêneo permite o tratamento da estrutura a termo do risco de crédito. Modelos mais complexos onde a intensidade é puramente estocástica, os processos de Cox (também chamados de duplamente estocásticos), são também usados. Neste caso, condicional à realização de um processo de intensidade exógeno dado $\lambda=\lambda_{t t \geq 0}$, para todo $s \leq t$, a contagem dos eventos aleatórios é dada por:

$$
\mathbb{P}\left[N_{t}-N_{s}=k \mid \lambda\right]=e^{-\int_{s}^{t} \lambda_{u} d u} \frac{\left(\int_{s}^{t} \lambda_{u} d u\right)^{k}}{k !}
$$

Se denotamos os tempos de ocorrência dos eventos aleatórios por $\tau_{1} \leq \tau_{2} \leq$ ..., então, $N_{t}=\sum_{j=1}^{\infty} \mathbf{1}_{\tau_{j} \leq t}$. Definimos agora o tempo de default $\tau$ como o tempo $\tau_{1}$ de primeira ocorrência do processo de contagem $N_{t}$, isto é:

Definição 1 O Tempo de default ou Evento de crédito é uma variável aleatória contínua $\tau$ assumindo valores em $(0,+\infty]$ definido como o tempo $\tau_{1}$ de primeira ocorrência do processo de contagem $N_{t}$, com $N_{0}=0$ e distribuição de probabilidades condicional:

$$
\mathbb{P}\left[N_{t}-N_{s}=k \mid \lambda\right]=e^{-\int_{s}^{t} \lambda_{u} d u} \frac{\left(\int_{s}^{t} \lambda_{u} d u\right)^{k}}{k !}
$$

Neste artigo vamos assumir que as realizações do processo de intensidade $\lambda$ são independentes das demais fontes de incerteza existentes no mercado. Assume-se, em particular, que o evento de crédito é independente das taxas de juros. Apesar de não muito realista, esta situação é extremamente atraente em virtude da facilidade com que podemos apreçar os derivativos de crédito concentrando-se apenas no risco de crédito.

Utilizando um processo de Poisson não-homogêneo a probabilidade de default de um título com vencimento em $T$ é dada pela seguinte equação:

$$
\mathbb{P}(\tau \leq T)=1-e^{-\int_{0}^{T} \lambda(t) d t}
$$

O processo $\lambda$ atua como uma medida da intensidade na qual uma empresa corre o risco de tornar-se inadimplente. Como num processo de Poisson não-homogêneo

\footnotetext{
${ }^{12}$ Ver em Lando (1998) uma introdução aos processos de Poisson com intensidade variável.
} 
$\lambda$ depende do tempo, tem-se que tal intensidade não é constante refletindo o fato que uma empresa não permanece no mesmo estado indefinidamente. Note que se $\lambda$ for alto por um longo período de tempo, a chance de ocorrer um default também torna-se alta.

Além da hipótese sobre o evento de crédito descrita acima, assume-se também que:

- os spreads são determinados unicamente em função do risco de crédito;

- o valor de recuperação em caso de default é determinado exógenamente.

Consideremos em princípio um título simples, isto é, sem pagamentos de cupons, e denotemos seu preço unitário por $D(t, T)$ caso haja risco de crédito e por $B(t, T)$, caso contrário.

$\mathrm{O}$ apreçamento de ativos financeiros com risco em uma economia sem oportunidades de arbitragem pode ser efeito através de medidas de probabilidade de Arrow-Debreu ${ }^{13}$ que fornecem o preço de mercado em termos dos valores esperados dos fluxos de caixa descontados. Enquanto que a não existência de oportunidades de arbitragem garante a existência de uma medida de Arrow-Debreu, sua unicidade depende da completitude da economia, mais precisamente, de completitude dinâmica. Vamos assumir neste caso, que nossa economia foi completada dinâmicamente negociando em qualquer instante de tempo, ativos com risco de crédito para quaisquer vencimentos. Neste caso, a medida de apreçamento será única, a qual denotaremos aqui por $\mathbb{Q}$.

Para um título de renda fixa não contendo risco de crédito, seu preço de mercado é:

$$
B(t, T)=\mathbf{E}_{t}^{\mathbb{Q}}\left[e^{-\int_{t}^{T} r_{s} d s}\right]
$$

onde $\mathbb{Q}$ é a medida de apreçamento de Arrow-Debreu e $\mathbf{E}_{t}[$.$] indica esperança$ condicional em relação a informação disponível até o instante $t$. No entanto, se há risco de crédito o preço do título é dado por:

$$
D(t, T)=\mathbf{E}_{t}^{\mathbb{Q}}\left[e^{-\int_{t}^{T} r_{s} d s} \mathbf{1}_{\{\tau>T\}}\right]
$$

onde $\mathbf{1}_{\tau>T}$ é a função indicadora do evento de sobrevida. Segue, então, da independência entre taxas de juros e evento de crédito que

$$
D(t, T)=\mathbf{E}_{t}^{\mathbb{Q}}\left[e^{-\int_{t}^{T} r_{s} d s}\right] \cdot \mathbf{E}_{t}^{\mathbb{Q}}\left[\mathbf{1}_{\{\tau>T\}}\right]=B(t, T) \cdot \mathbb{P}[\tau>T \mid \tau>t]
$$

Aqui, $\mathbb{P}[\tau>T \mid \tau>t]$ denota a probabilidade de que o evento de crédito não ocorra antes do prazo de vencimento do título $T$ dado que até o momento $t$

${ }^{13}$ Risk-Neutral Measure ou "medida ajustada ao risco". 
a empresa ainda não havia entrado em default. Tal probabilidade é denominada probabilidade de sobrevida. Por outro lado,

$$
\mathbb{P}[\tau \leq T \mid \tau>t]=1-\mathbb{P}[\tau>T \mid \tau>t]
$$

chama-se probabilidade acumulada de default.

Note que até o momento não temos considerado a possibilidade de recuperação, ou melhor. Por hipótese, o valor de recuperação no caso de default é uma variável exógena. Vamos considerar dois casos, o primeiro onde a recuperação ocorre no vencimento do título e uma fração $\delta$ do principal é recuperada e um segundo caso onde a recuperação é feita no momento incerto do default e o valor recuperado é uma fração $\delta$ do valor de mercado imediatamente antes do instante de default.

No primeiro caso, com recuperação no instante do vencimento, temos que:

$$
D(t, T)=\mathbf{E}_{t}^{\mathbb{Q}}\left[e^{-\int_{t}^{T} r_{s} d s}\left(\mathbf{1}_{\tau>T}+\delta \mathbf{1}_{\{\tau \leq T\}}\right)\right]
$$

onde $\mathbf{E}_{t}^{\mathbb{Q}}\left[e^{-\int_{t}^{T} r_{s} d s} \mathbf{1}_{\tau>T}\right]$ representa o pagamento do principal caso não haja default, e $\mathbf{E}_{t}^{\mathbb{Q}}\left[e^{-\int_{t}^{T} r_{s} d s} \delta \mathbf{1}_{\tau \leq T}\right]$ representa o valor da recuperação em caso de default. Então, repetindo o mesmo procedimento usado na equação (18) tem-se que:

$$
D(t, T)=B(t, T) \cdot\{\delta+(1-\delta) \mathbb{P}[\tau>T \mid \tau>t]\}
$$

Para o caso de recuperação no instante do default, a situação é um pouco mais complicada em virtude do valor justo estar implícito na relação:

$$
\left.D(t, T)=\mathbf{E}_{t}^{\mathbb{Q}}\left[e^{-\int_{t}^{T} r_{s} d s} \mathbf{1}_{\tau>T}+\delta e^{-\int_{t}^{\tau} r_{s} d s} D(\tau-, T) \mathbf{1}_{\tau \leq T}\right)\right]
$$

Para isolarmos o valor justo $D(t, T)$ é preciso manipular a equação (22). Segue da fórmula (20) que o risco de crédito pode ser expresso através de uma taxa de desconto, $R$, que incorpora o risco de default. O próximo teorema, devido à Duffie (1999) que formaliza essa questão:

Teorema $1 O$ valor justo de mercado $D(t, T)$ de um título de renda fixa com risco de crédito e recuperação do valor de mercado no momento do default é dado por:

$$
D(t, T)=\mathbf{E}_{t}^{\mathbb{Q}}\left[e^{-\int_{t}^{T} R_{s} d s}\right]
$$

onde a taxa de desconto ajustada ao risco de crédito é dada por $R_{t}:=r_{t}+\lambda_{t}(1-$ $\delta)$.

Prova. No caso geral, pode-se obter em Duffie e Singleton (1999) a prova do teorema. No entanto, assumindo independência dos fatores de risco de juros e 
crédito, obtemos uma versão simplificada da demonstração. Seja $V_{t}=D(t, T)$ o valor justo do título em $t$. Neste caso:

$$
\begin{aligned}
V_{t} & \left.=\mathbf{E}_{t}^{\mathbb{Q}}\left[e^{-\int_{t}^{T} r_{s} d s} \mathbf{1}_{\{\tau>T\}}+\delta e^{-\int_{t}^{\tau} r_{s} d s} V_{\tau-} \mathbf{1}_{\{\tau \leq T\}}\right)\right] \\
& =\mathbf{E}_{t}^{\mathbb{Q}}\left[\mathbf{E}_{t}^{\mathbb{Q}}\left[e^{-\int_{t}^{T} r_{s} d s} \mathbf{1}_{\{\tau>T\}} \mid r\right]\right] \\
& +\delta \mathbf{E}_{t}^{\mathbb{Q}}\left[\mathbf{E}_{t}^{\mathbb{Q}}\left[e^{-\int_{t}^{\tau} r_{s} d s} V_{\tau-} \mathbf{1}_{\{\tau \leq T\}} \mid r\right]\right] \\
& =\mathbf{E}_{t}^{\mathbb{Q}}\left[e^{-\int_{t}^{T} r_{s} d s} \mathbf{E}_{t}^{\mathbb{Q}}\left[\mathbf{1}_{\tau>T} \mid\{r\}\right]\right] \\
& \left.+\delta \mathbf{E}_{t}^{\mathbb{Q}}\left[\mathbf{E}_{t}^{\mathbb{Q}}\left[e^{-\int_{t}^{\tau} r_{s} d s} V_{\tau-} \mathbf{1}_{\{\tau \leq T\}}\right) \mid\{r\}\right]\right]
\end{aligned}
$$

Assumindo a independência das taxas de juros e do evento de crédito, temos que:

$$
\mathbf{E}_{t}^{\mathbb{Q}}\left[\mathbf{1}_{\tau>T} \mid\{r\}\right]=\mathbf{E}_{t}^{\mathbb{Q}}\left[\mathbf{1}_{\{\tau>T\}}\right]=e^{-\int_{t}^{T} \lambda_{s} d s}
$$

Assim, o valor justo $V_{t}$ pode ser escrito como:

$$
\left.V_{t}=\mathbf{E}_{t}^{\mathbb{Q}}\left[e^{-\int_{t}^{T}\left(r_{s}+\lambda_{s}\right) d s}\right]+\delta \mathbf{E}_{t}^{\mathbb{Q}}\left[\mathbf{E}_{t}^{\mathbb{Q}}\left[e^{-\int_{t}^{\tau} r_{s} d s} V_{\tau-} \mathbf{1}_{\{\tau \leq T\}}\right) \mid\{r\}\right]\right]
$$

Para calcularmos o segundo termo, assumimos novamente que a variável $\lambda$ é independente dos valores futuros das taxas de juros $\left\{r_{s}\right\}_{t<s<T}$ e, portanto,

$$
\left.\mathbf{E}_{t}^{\mathbb{Q}}\left[e^{-\int_{t}^{\tau} r_{s} d s} V_{\tau-} \mathbf{1}_{\{\tau \leq T\}}\right) \mid\{r\}\right]=\int_{t}^{T} e^{-\int_{t}^{s} r_{u} d u} V_{s} \lambda_{s} e^{-\int_{t}^{s} \lambda_{u} d u}
$$

onde fizemos uso do fato que a variável $\tau$ tem $\lambda_{s} e^{-\int_{t}^{s} \lambda_{u} d u}$ como densidade de probabilidade condicional. Assim, o valor $V_{t}$ pode ser finalmente escrito como:

$$
V_{t}=\mathbf{E}_{t}^{\mathbb{Q}}\left[e^{-\int_{t}^{T}\left(r_{s}+\lambda_{s}\right) d s}\right]+\delta \mathbf{E}_{t}^{\mathbb{Q}}\left[\int_{t}^{T} e^{-\int_{t}^{s}\left(r_{u}+\lambda_{u}\right) d u} V_{s} \lambda_{s} d s\right]
$$

Para provarmos o resultado, é conveniente introduzirmos o processo $M_{t}$, dado por:

$$
M_{t}=\mathbf{E}_{t}^{\mathbb{Q}}\left[e^{-\int_{0}^{T}\left(r_{s}+\lambda_{s}\right) d s}+\delta \int_{0}^{T} e^{-\int_{0}^{s}\left(r_{u}+\lambda_{u}\right) d u} V_{s} \lambda_{s} d s\right]
$$

Como $M_{t}$ é um processo filtrado, isto é, um processo da forma $M_{t}=\mathbf{E}_{t}^{\mathbb{Q}}[Y]$, então é um Martingal também. Usaremos este fato de modo crucial adiante. Reescrevendo o valor de $V_{t}$, temos que: 


$$
\begin{aligned}
V_{t} & =\mathbf{E}_{t}^{\mathbb{Q}}\left[e^{-\int_{t}^{T}\left(r_{s}+\lambda_{s}\right) d s}+\delta \int_{t}^{T} e^{-\int_{t}^{s}\left(r_{u}+\lambda_{u}\right) d u} V_{s} \lambda_{s} d s\right] \\
& =e^{\int_{0}^{t}\left(r_{s}+\lambda_{s}\right) d s} e^{-\int_{0}^{t}\left(r_{s}+\lambda_{s}\right) d s} \mathbf{E}_{t}^{\mathbb{Q}}\left[e^{-\int_{t}^{T}\left(r_{s}+\lambda_{s}\right) d s}\right. \\
& \left.+\delta \int_{t}^{T} e^{-\int_{t}^{s}\left(r_{u}+\lambda_{u}\right) d u} V_{s} \lambda_{s} d s\right] \\
& =e^{\int_{0}^{t}\left(r_{s}+\lambda_{s}\right) d s}\left(M_{t}-\delta \int_{0}^{t} e^{-\int_{0}^{s}\left(r_{u}+\lambda_{u}\right) d u} V_{s} \lambda_{s} d s\right)
\end{aligned}
$$

Definindo $Z_{t}:=e^{\int_{0}^{t}\left(r_{s}+\lambda_{s}\right) d s}$, podemos, com o auxílio do lema de Itô, obter a seguinte expressão para a derivada estocástica $d Z_{t}$ :

$$
\begin{aligned}
d V_{t} & =Z_{t} d M_{t}+\left(r_{t}+\lambda_{t}\right) Z_{t} M_{t} d t-\delta Z_{t} e^{-\int_{0}^{t}\left(r_{u}+\lambda_{u}\right) d u} V_{t} \lambda_{t} d t \\
& -\delta Z_{t}\left(r_{t}+\lambda_{t}\right) d t \int_{0}^{t} e^{-\int_{0}^{s}\left(r_{u}+\lambda_{u}\right) d u} V_{s} \lambda_{s} d s \\
& =Z_{t} d M_{t}+\left(r_{t}+\lambda_{t}\right) Z_{t} M_{t} d t-\delta V_{t} \lambda_{t} d t-\left(r_{t}+\lambda_{t}\right)\left(M_{t} Z_{t}-V_{t}\right) d t \\
& =\left(r_{t}+(1-\delta) \lambda_{t}\right) V_{t} d t+Z_{t} d M_{t}
\end{aligned}
$$

Denotando a taxa efetiva por $R_{t}:=r_{t}+\lambda_{t}(1-\delta)$, podemos integrar a equação estocástica $d V=R V d t+Z d M$ entre $t$ e $T$ para obter o resultado desejado, usando o fato que o processo $M_{t}$ é um martingal e portanto o termo proveniente da integral estocástica com relação à $d M_{t}$ têm valor esperado nulo.

Para aplicarmos os modelos de intensidade à situação de mercado, introduzimos os retornos dos títulos com e sem risco de crédito, isto é, $D(0, T)=e^{-y_{T} T}$, $B(0, T)=e^{-y_{T}^{0} T}$ e isolamos as probabilidades implícitas de default nos spreads de crédito $s_{T}=y_{T}-y_{T}^{0}$. Para o modelo com recuperação no vencimento, consideramos a probabilidade acumulada de default na equação (21) e obtemos:

$$
\mathbb{P}(\tau \leq T)=\frac{1}{1-\delta}\left(1-e^{-s_{T} T}\right)
$$

Para o caso da recuperação no momento de default, obtemos uma expressão semelhante.

O uso dos modelos de intensidade se torna atrativo para uso em mercados em virtude da flexibilidade de parametrização da estrutura a termo de default, quer dizer, da estrutura temporal da intensidade de default $\lambda=\left\{\lambda_{t}\right\}_{t>0}$. Apesar de teoricamente ser possível parametrizarmos a estrutura a termo de forma a permitir dependências com fatores de risco macroeconômico como taxas de juros, vamos assumir uma estrutura simples onde $\lambda=\left\{\lambda_{t}\right\}_{t \geq 0}$, é não somente determinístico mas também constante por partes. Para isto, vamos parametrizar a estrutura de 
risco de crédito em vértices fixos coincidentes com os vencimentos $0=T_{0}<$ $T_{1}<T_{2}<\ldots<T_{N}=T$ de $N$ títulos privados do mesmo emissor, e impor que $\lambda$ assuma o valor constante $\lambda_{i}$ entre os vencimentos $T_{i-1}$ e $T_{i}$ para $i=1, \ldots, N$.

Os spreads de crédito fornecem estimativas para as probabilidades de sobrevida $\mathbb{P}(\tau \leq T)$, ou, equivalentemente, para as intensidades acumuladas $\Lambda_{i}=$ $\int_{0}^{T_{i}} \lambda_{s} d s$. As intensidades são obtidas recursivamente através da equação:

$$
\lambda_{i}=\frac{\Lambda_{i}-\Lambda_{i-1}}{T_{i}-T_{i-1}}
$$

Isto segue do fato que:

$$
\Lambda_{i}-\Lambda_{i-1}=\int_{T_{i-1}}^{T_{i}} \lambda_{i} d s=\lambda_{i}\left(T_{i}-T_{i-1}\right)
$$

de onde resulta a equação (33).

Como a determinação do processo de intensidade $\lambda$ é obtido a partir dos spreads observados no mercado faz-se necessária a checagem de possíveis inconsistências no modelo. ${ }^{14} \mathrm{O}$ teorema abaixo explicita a condição suficiente para que o modelo seja consistente, quer dizer, as probabilidades de default extraídas do mercado sejam todas não-negativas.

Teorema 2 Condição de Consistência. Se para todo $i=1, \ldots, N$ os spreads de crédito verificam as condições:

$$
\frac{s_{T_{i-1}}}{s_{T_{i}}} \leq \frac{T_{i}}{T_{i-1}}
$$

então, o modelo de intensidade parametrizado de forma constante por partes é consistente com os spreads de mercado.

Prova. Das hipóteses do teorema tem-se que

$$
s_{T_{i-1}} T_{i-1} \leq s_{T_{i}} T_{i}
$$

e, portanto,

$$
e^{-s_{T_{i}} T_{i}} \leq e^{-s_{T_{i-1}} T_{i-1}}
$$

Logo,

\footnotetext{
${ }^{14} \mathrm{~A}$ existência de inconsistências no modelo implica em uma função de probabilidade acumulada decrescente em algum intervalo do seu domínio.
} 


$$
\begin{aligned}
\mathbb{P}\left(\tau \leq T_{i-1}\right) & =\frac{1}{1-\delta}\left(1-e^{-s_{T_{i-1}} T_{i-1}}\right) \\
& \leq \frac{1}{1-\delta}\left(1-e^{-s_{T_{i}} T_{i}}\right)=\mathbb{P}\left(\tau \leq T_{i}\right)
\end{aligned}
$$

o que demonstra o teorema.

Embora o argumento acima tenha sido puramente matemático, a condição (34) tem um significado econômico. O spread de uma emissão mais longa não pode ser arbitrariamente menor que o spread do vértice anterior, pois do contrário uma "probabilidade" negativa para o período seria gerada. Por outro lado, se os spreads obtidos no mercado são compatíveis com o modelo, então, é possível construir recursivamente a estrutura a termo da intensidade ${ }^{15} \lambda$.

\subsection{Modelos de rating}

Um modelo de rating classifica as mais diversas instituições (países, empresas, etc.) de acordo com seu risco de inadimplência. Essa classificação é feita via conceitos como, por exemplo:

- $1,2, \ldots, N$, onde 1 representa um risco extremamente baixo de inadimplência, enquanto que $N$ representa o estado de default;

- $A A A, A A, A, B A, B, B B, \ldots, D$, onde $A A A$ é o melhor conceito e $D$ é o estado de default.

Estes conceitos são conhecidos por ratings e a eles estão associadas a faixas de probabilidades de um evento de crédito. A especificação dessas faixas é proprietária, ou seja, são determinadas pelas agências classificadoras de risco de crédito.

Vamos supor que os ratings estejam ordenados de 1 a $N$, onde 1 é o nível menos arriscado (melhor conceito) e $N$ representa o próprio estado de default. Nesse caso, $N$ é um estado absorvente. Uma outra característica empírica, é que quanto pior o rating de um certo título maior deverá ser seu spread.

Dentre os modelos de rating destacamos o modelo de Jarrow et alii (1997). Nesse modelo o tempo de default é modelado estocasticamente através de uma matriz de transição de ratings:

$$
P(t, s)=\left(P_{i j}(t, s)\right)_{i j}
$$

onde $i, j=1, \ldots, N$ e $P_{i j}(t, s)$ é a probabilidade de um título com rating $i$ no instante $t$ obter um rating $j$ no instante $s$. Obviamente, $P_{i j} \geq 0$ e $\sum_{j} P_{i j}=1 \mathrm{e}$ como estamos assumindo que $N$ é um estado absorvente tem-se que $P_{N N}=1$. As

${ }^{15}$ Através das expressões (15), (32) e (33), obtém-se recursivamente a estrutura a termo de $\lambda$. 
matrizes $P(t, s)$ formam um semi-grupo, isto é, para todo $t<s<T, P(t, T)=$ $P(t, s) P(s, T)$. Em termos de seus elementos, isto é expresso pela equação de Chapman-Kolmogorov:

$$
P_{i j}(t, T)=\sum_{k=1}^{N} P_{i k}(t, s) P_{k j}(s, T)
$$

Além disto, assumimos as hipóteses adicionais:

- as probabilidades $P_{i j}$ são estacionárias

$$
P_{i j}(t, s)=P_{i j}(s-t)
$$

ou seja, as probabilidades de transição não dependem dos instantes $t$ e $s$ mas da distância entre ambos;

- as matrizes de transição são ajustadas ao risco.

Considerar processos estacionários é uma simplificação do mundo real, no entanto, ainda é uma boa aproximação. Mais ainda, permite a definição de benchmarks de crédito. Para construir as matrizes de transição consideramos, em princípio, a matriz de ratings para 1 ano $P(1)$. A partir dela obtemos o gerador infinitesimal $\Lambda$ :

$$
P(1)=e^{\Lambda}
$$

da qual pode-se construir as demais matrizes de transição. ${ }^{16}$ Tendo obtido as matrizes de transição e, consequentemente, as probabilidades de default para os diversos ratings, pode-se calcular os preços dos títulos com risco de crédito através da fórmula (21).

Deve-se, no entanto, observar que dependendo da matriz de ratings, o gerador infinitesimal pode não existir ou, se existir, não possuir as propriedades corretas. Os problemas que podem ocorrer são basicamente os seguintes:

- gerador inexistente no mundo real;

- autovalores complexos;

- violação da condição de positividade;

- "probabilidades" negativas.

\footnotetext{
${ }^{16}$ Segue da equação de Chapman-Kolmogorov que $\mathbb{P}$ deve satisfazer ao sistema de equações diferenciais ordinárias $\dot{\mathbb{P}}(t)=\mathbb{P}(t) \Lambda=\Lambda \mathbb{P}(t)$ a partir do qual tem-se que $\mathbb{P}(\Delta t)=\exp (\Lambda \cdot \Delta t)$.
} 
Dizemos que um gerador infinitesimal está bem-definido se a matriz $P(1)$ satisfizer a seguinte condição: ${ }^{17}$

$$
\left|P_{i i}-1\right|<\frac{1}{2}
$$

Ou seja, a probabilidade de permanência no mesmo estado deve ser superior a $\frac{1}{2}$.

Finalmente, com a matriz de transição nas mãos podemos apreçar títulos de crédito exatamente do mesma maneira que na seção anterior, bastando para isso observar que a probabilidade de default corresponde à última coluna da matriz de transição.

\subsubsection{Um exemplo}

Considere uma situação na qual existem apenas três estados de crédito:

- Estado 1: muito bom;

- Estado 2: regular;

- Estado 3: default.

De acordo com o modelo o estado 3 deve ser um estado absorvente, pois estamos assumindo que não há como sair do estado de default. Uma possível matriz de transição para um período de um ano, por exemplo, seria

\begin{tabular}{|c|c|c|c|}
\hline \multicolumn{4}{|c|}{$\begin{array}{l}\text { Exemplo de uma matriz de transição } \\
\text { em um mundo com apenas três estados }\end{array}$} \\
\hline$\overline{\mathbb{P}}$ & 1 & 2 & 3 \\
\hline 1 & 0.95 & 0.03 & 0.02 \\
\hline 2 & 0.15 & 0.70 & 0.15 \\
\hline 3 & 0.00 & 0.00 & 1.00 \\
\hline
\end{tabular}

De acordo com a matriz de transição acima um título com classificação 1, ou seja, muito bom, apresenta um risco de default de apenas $2 \%$, enquanto que a probabilidade de que ele permaneça nesse nível é alta, de $95 \%$. Também é fácil verificar que essa matriz de transição satisfaz a condição (36) e que, consequentemente, existe um gerador infinitesimal. Desse modo, a partir dela é possível obter as demais matrizes de transição para outros períodos de tempo.

A vantagem do uso dos modelos de ratings no caso da não existem emissões de títulos de renda fixa para vários vencimentos é que podemos, a partir do gerador infinitesimal, ajustar as probabilidades de default para um vencimento qualquer. Uma vez conseguidas estas probabilidades, o apreçamento do título de renda fixa é imediato. Para um título de renda fixa com vencimento em $T$, rating $i$, e recuperação no vencimento, obtemos para o seu valor $D_{i}(t, T)$ a seguinte expressão:

$$
D_{i}(t, T)=B(t, T) \cdot\left\{\delta+(1-\delta) \mathbb{P}\left[\tau_{i}>T \mid \tau_{i}>t\right]\right\}
$$

\footnotetext{
${ }^{17}$ Ver Israel et alii (2001)
} 
onde $\tau_{i}$ é o instante de default de um título com rating igual a $i$ e $\mathbb{P}\left[\tau_{i}>T \mid \tau_{i}>t\right]$ é sua probabilidade de sobrevida até o instante $T$.

Se objetivamos apreçar um derivativo de crédito, então as probabilidades de default já são suficientes para este fim.

\section{Derivativos de crédito - Credit Default Swap}

Diferentemente dos derivativos usuais nos quais os ativos base são ações, futuros, índices, opções, etc., nos derivativos de crédito o ativo subjacente é o crédito a uma terceira parte. Ou seja, duas partes negociam o risco de crédito que uma delas assumiu em outra ocasião com uma terceira parte. Nesse acordo, uma das partes transfere seu risco assumido no momento da concessão do crédito a um certo custo. Existem diversas modalidades de derivativos e estruturas de crédito sendo negociados no mercado internacional:

- swap de crédito;

- swap de retorno total;

- opções de crédito;

- notas ligadas ao crédito;

- collaterized obligations - CO's

$$
\begin{aligned}
& \circ \mathrm{CDO}-d e b t \\
& \circ \mathrm{CLO}-\text { loan. }
\end{aligned}
$$

Neste artigo nos focamos no swap de crédito (ou Credit Default Swap).

A dinâmica do swap de crédito é muito simples. Suponha que uma empresa $E$ tomou um crédito de um credor $C$ (por exemplo, um banco). Esse crédito pode ter sido obtido com a venda de debêntures, por exemplo. Ora, então, $C$ assumiu um risco, pois existe a possibilidade de inadimplência. Para se proteger, $C$ recorre a um vendedor de hedge $H$ (seguro) que em troca de uma taxa de proteção pré-fixada assume o risco de crédito, como pode ser visto na figura 1.

Nosso principal interesse reside no cálculo da taxa de proteção. ${ }^{18} \mathrm{O}$ modo de pagamento dessa taxa de proteção é variado, podendo ser pago à vista ou em parcelas. Na próxima seção mostraremos como calcular tal taxa utilizando um modelo de intensidade.

\footnotetext{
${ }^{18}$ Ver em Hull e White (2000) e Schonbucher (2000) definições e descrições dos swaps de crédito
} 


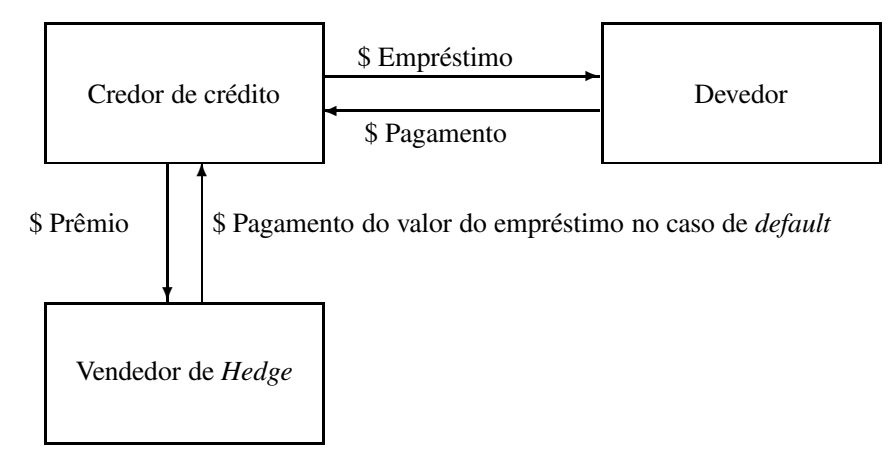

Figura 1

Credit Default Swap

\section{Apreçando um Swap de Crédito no Mercado}

O objetivo dessa seção consiste na aplicação de um método para avaliação do risco de crédito no apreçamento de instrumentos de crédito no mercado brasileiro. Mais precisamente, mostraremos como usar o modelo de intensidade descrito acima para calcular o valor da taxa de proteção de um swap de crédito. Vamos assumir nesta seção que o processo de intensidade de default é determinístico para simplificar os cálculos.

Os fluxos de caixa em um swap de crédito são compostos de

- pagamentos da taxa de proteção;

- pagamento do valor protegido em caso de default.

Vamos denotar a taxa de proteção por $C D S$ e o tempo de default por $\tau$. Então, o valor atualizado dos pagamentos da taxa de proteção nas datas especificadas no contrato é dado por:

$$
-\mathbf{E}_{t}^{\mathbb{Q}}\left[\sum_{j=1}^{m} C D S \cdot \mathbf{1}_{\tau>t_{j}} e^{-\int_{t}^{t_{j}} r_{s} d s}\right]
$$

enquanto que o pagamento do valor protegido, em caso de default, é dado por

$$
\mathbf{E}_{t}^{\mathbb{Q}}\left[\mathbf{1}_{\tau \leq T}(1-\delta) e^{-\int_{t}^{T} r_{s} d s}\right]
$$

no caso em que a recuperação ocorre na data de vencimento, ou por 


$$
\mathbf{E}_{t}^{\mathbb{Q}}\left[\mathbf{1}_{\tau \leq T}(1-\delta) e^{-\int_{t}^{\tau} r_{s} d s}\right]
$$

no caso onde a recuperação ocorre no momento do default.

O valor justo do swap de crédito, $C D S$, assumindo o esquema de recuperação implícito na equação (40), é obtido igualando-se os fluxos de caixa do instrumento no momento da avaliação do contrato:

$$
\mathbf{E}_{t}^{\mathbb{Q}}\left[\sum_{j=1}^{m} C D S \cdot \mathbf{1}_{\tau>t_{j}} e^{-\int_{t}^{t_{j}} r_{s} d s}\right]=\mathbf{E}_{t}^{\mathbb{Q}}\left[1_{\tau \leq T}(1-\delta) e^{-\int_{t}^{\tau} r_{s} d s}\right]
$$

e, a seguir, isolando seu valor

$$
\begin{aligned}
C D S & =\frac{\mathbf{E}_{t}^{\mathbb{Q}}\left[\mathbf{1}_{\{\tau \leq T\}}(1-\delta) e^{-\int_{t}^{\tau} r_{s} d s}\right]}{\mathbf{E}_{t}^{\mathbb{Q}}\left[\sum_{j=1}^{m} 1_{\left\{\tau>t_{j}\right\}} e^{-\int_{t}^{t_{j}} r_{s} d s}\right]} \\
& =(1-\delta) \frac{\mathbf{E}_{t}^{\mathbb{Q}}\left[\mathbf{1}_{\{\tau \leq T\}} e^{-\int_{t}^{\tau} r_{s} d s}\right]}{\sum_{j=1}^{m} B\left(t, t_{j}\right) \mathbf{E}_{t}^{\mathbb{Q}}\left[\mathbf{1}_{\left\{\tau>t_{j}\right\}}\right]}
\end{aligned}
$$

onde $B(.,$.$) é o preço de um título zero cambial sem risco de crédito. Segue daí$ que

$$
C D S=(1-\delta) \frac{\int_{t}^{T} \lambda_{s} e^{-\int_{t}^{s} \lambda_{u} d u} B(t, s) d s}{\sum_{j=1}^{m} B\left(t, t_{j}\right) \mathbb{P}\left(\tau>t_{j}\right)}
$$

sendo $\lambda$ o processo de intensidade descrito na subseção 2.2.

No caso em que a recuperação ocorre na data de vencimento tem-se que

$$
C D S=(1-\delta) \frac{B(t, T) \mathbb{P}(\tau \leq T)}{\sum_{j=1}^{m} B\left(t, t_{j}\right) \mathbb{P}\left(\tau>t_{j}\right)} .
$$

De fato, se o membro a direita na equação (41) é substituido por (39), tem-se isolando a variável $C D S$ que

$$
\begin{aligned}
C D S & =\frac{\mathbf{E}_{t}^{\mathbb{Q}}\left[\mathbf{1}_{\tau \leq T}(1-\delta) e^{\left.-\int_{t}^{T} r_{s} d s\right]}\right.}{\mathbf{E}_{t}^{\mathbb{Q}}\left[\sum_{j=1}^{m} \mathbf{1}_{\left\{\tau>t_{j}\right\}} e^{-\int_{t}^{t_{j}} r_{s} d s}\right]} \\
& =(1-\delta) \frac{e^{-\int_{t}^{T} r_{s} d s} \mathbf{E}_{t}^{\mathbb{Q}}\left[\mathbf{1}_{\{\tau \leq T\}}\right]}{\sum_{j=1}^{m} B\left(t, t_{j}\right) \mathbf{E}_{t}^{\mathbb{Q}}\left[\mathbf{1}_{\left\{\tau>t_{j}\right\}}\right]} \\
& =(1-\delta) \frac{B(t, T) \mathbb{P}(\tau \leq T)}{\sum_{j=1}^{m} B\left(t, t_{j}\right) \mathbf{E}_{t}^{\mathbb{Q}}\left[\mathbf{1}_{\left\{\tau>t_{j}\right\}}\right]}
\end{aligned}
$$


onde foram utilizadas as identidades $B(t, T)=\mathbf{E}_{t}^{\mathbb{Q}}\left[e^{-\int_{t}^{T} r_{s} d s}\right]$ e $\mathbb{P}(\tau \leq T)=$ $\mathbf{E}_{t}^{\mathbb{Q}}\left[\mathbf{1}_{\{\tau \leq T\}}\right]$.

\section{Uma Aplicação: Títulos Cambiais}

Nesta seção ilustramos como podemos apreçar um swap de crédito que protege um particular título cambial emitido pelo tesouro. Utiliza-se nesta aplicação o modelo de intensidade para o apreçamento do CDS, representado pela equação (43), onde a recuperação ocorre no vencimento do título. Como exemplo, tomaremos as stripped NTN's Cambiais (sintéticas sem cupons), que na verdade são instrumentos cambiais zero coupon. Suponhamos que estejamos analisando tais títulos no dia 13/02/2003 e que tenhamos a situação de mercado representada nas Tabelas 1 e 2 . Vale a pena observar que as taxas contínuas diárias livre de risco na tabela foram obtidas a partir dos vértices da curva de cupom cambial divulgada pela BM\&F (Linear/360). Além disso, assumiremos uma taxa de recuperação $\delta=20 \%$ e que os pagamentos da taxa de proteção se darão trimestralmente.

Tabela 1

Cupom das stripped cambiais

\begin{tabular}{lcccc}
\hline \multicolumn{1}{c}{ Vencimentos } & $17 / 04 / 03$ & $18 / 12 / 03$ & $14 / 10 / 04$ & $13 / 10 / 05$ \\
\hline Dias Corridos & 63 & 308 & 609 & 973 \\
Cupom Stripped NTN Cambial & $11,0082 \%$ & $18,0000 \%$ & $23,0000 \%$ & $25,0000 \%$ \\
Cupom Cambial Livre de Risco & $9,5230 \%$ & $16,6945 \%$ & $18,8873 \%$ & $19,8596 \%$ \\
\hline
\end{tabular}

Tabela 2

Taxas contínuas diárias

\begin{tabular}{lcccc}
\hline \multicolumn{1}{c}{ Vencimentos } & $17 / 04 / 03$ & $18 / 12 / 03$ & $14 / 10 / 04$ & $13 / 10 / 05$ \\
\hline Dias Corridos & 63 & 308 & 609 & 973 \\
Taxas Stripped NTN Cambial & $0,0303 \%$ & $0,0465 \%$ & $0,0540 \%$ & $0,0531 \%$ \\
Taxas Cambial Livre de Risco & $0,0262 \%$ & $0,0433 \%$ & $0,0455 \%$ & $0,0442 \%$ \\
Spreads & $0,0041 \%$ & $0,0032 \%$ & $0,0084 \%$ & $0,0089 \%$ \\
\hline
\end{tabular}

A partir dos spreads e dos períodos para o vencimento realizamos o teste de consistência (34) e obtemos os resultados da Tabela 5, de onde concluimos (ver Teorema 2) que a implementação do modelo de intensidade conforme descrito na seção 2 pode ser aplicada a esse caso. Assim como para o teste de consistência, os preços das stripped NTN's cambiais e zeros cambiais sintéticos livre de risco são obtidos a partir das taxas contínuas na Tabela 2.

Tabela 3

Teste de consistência

\begin{tabular}{llll}
\hline Vencimentos & $17 / 04 / 03$ & $18 / 12 / 03$ & $14 / 10 / 04$ \\
\hline Teste & $26,253 \%$ & $18,928 \%$ & $59,368 \%$ \\
\hline
\end{tabular}


Tabela 4

Preço e probabilidades de sobrevida

\begin{tabular}{ccccc}
\hline Vencimentos & $17 / 04 / 03$ & $18 / 12 / 03$ & $14 / 10 / 04$ & $13 / 10 / 05$ \\
\hline Stripped NTN Cambial & 0,9810997 & 0,8665511 & 0,7198992 & 0,5967675 \\
Preço do zero livre de risco & 0,9836079 & 0,8750203 & 0,7578567 & 0,6507187 \\
Probabilidade de Sobrevida & $99,68 \%$ & $98,79 \%$ & $93,74 \%$ & $89,64 \%$ \\
\hline
\end{tabular}

As probabilidades de sobrevida são calculadas de acordo com a fórmula (21), usando a taxa de recuperação $\delta=20 \%$. Em nosso caso concreto temos as probabilidades de default apresentadas na tabela 5 .

Tabela 5

Probabilidades de default

\begin{tabular}{ccccc}
\hline Vencimentos & $17 / 04 / 03$ & $18 / 12 / 03$ & $14 / 10 / 04$ & $13 / 10 / 05$ \\
\hline Prob. de default & $0,3188 \%$ & $1,2099 \%$ & $6,2607 \%$ & $10,3638 \%$ \\
\hline
\end{tabular}

Com as probabilidades de default podemos apreçar esse instrumento de crédito de acordo com o modelo de intensidade. Para isso, basta aplicarmos a fórmula (43):

$$
C D S=(1-\delta) \frac{B(t, T) \mathbb{P}(\tau \leq T)}{\sum_{j=1}^{m} B\left(t, t_{j}\right) \mathbb{P}\left(\tau>t_{j}\right)}
$$

onde $B\left(t, t_{j}\right)$ representa o fator de desconto para os períodos intermediários de pagamentos do swap e $B(t, T)$, por sua vez, sendo o fator de desconto para o período total do swap. Logo,

$$
C D S=(1-0,20) \frac{0,067439}{8,313215}=0,6490 \%
$$

O valor porcentual acima representa o pagamento do swap de crédito trimestral que iguala o valor presente esperado do sinistro de crédito ao fluxo esperado dos pagamentos de swap na equação (41). Desse modo, exemplificamos como é possível apreçar um swap de crédito para um instrumento de renda fixa do mercado financeiro brasileiro, utilizando o modelo de intensidade.

\section{Conclusões}

Nesse artigo foram vistas as derivações do preço de uma obrigação de crédito, segundo os modelos estruturais, de intensidade e de ratings. Devido a baixa liquidez dos mercados financeiros corporativos no Brasil e do reduzido universo de empresas locais às quais uma aplicação dos modelos estruturais poderia ser tentada, outras abordagens parecem ser mais indicadas. Além disto, como salientamos na subseção 2.1 inúmeros problemas metodológicos e empíricos prejudicam a performance desta classe de modelos. Notamos ainda que no Brasil e em outros países, títulos soberanos exibem risco de crédito, o que de imediato dificultaria ainda mais o uso dos modelos estruturais no apreçamento destes ativos. 
Os modelos de intensidade, enquanto tradutores de mercados líquidos, dependem quase que exclusivamente de sua existência, sendo sua utilização no Brasil limitada às pequenas ilhas de liquidez existentes nos mercados de títulos de renda fixa locais. Esta situação específica foi explorada na aplicação desenvolvida na subseção 2.2 deste artigo.

Os modelos de rating, por sua vez, dependem de parâmetros que melhor se adequam à natureza do nosso mercado de crédito. Vale lembrar que a inexistência de liquidez e preços de mercado para os títulos de crédito locais, fazem com que os modelos de rating produzam preços de referência ou de arbitragem.

Finalmente, consideramos o apreçamento de um swap de crédito em geral e uma aplicação prática para uma NTN cambial sintética (sem cupom) segundo modelos de intensidade.

\section{Referências}

Belangier, A., Schreve, S., \& Wong, D. (2001). A unified model for credit derivatives. Carnegie Mellon. Working Paper.

Bielecki, T. R. \& Rutkowski, M. (2002). Credit Risk: Modeling, Valuation and Hedging. Springer-Verlag, Berlin, $1^{a}$ edition.

Black, F. \& Cox, J. C. (1976). Valuing corporate securities: Some effects of bond indenture provisions. Journal of Finance, 31:351-367.

Black, F. \& Scholes, M. (1973). The pricing of options and corporate liabilities. Journal of Political Economy, 81:637-654.

Bohn, J. (1999). A survey of contingent-claims approaches to risky debt valuation. Haas School of Business, University of California, Berkeley. Working Paper.

Brys, E. \& De Varenne, F. (1997). Valuing risky fixed rate debt: An extension. Journal of Financial and Quantitative Analysis, 32:239-248.

Cossin, D. \& Pirotte, H. (2000). Advanced Credit Risk Analysis. John Wiley \& Sons, New York, $1^{a}$ edition.

Duffie, D. (1996). Dynamic Asset Pricing. Princeton University Press, Princeton, $2^{a}$ edition.

Duffie, D. (1999). Credit swap valuation. Financial Analysis Journal, 55(1):7387.

Duffie, D. \& Singleton, K. (1999). Modelling term structures of defaultable bonds. Rev. Finan. Stud., 12:687-720.

Ericsson, J. \& Reneby, J. (1998). A framework for valuing corporate securities. Applied Mathmatics Finance, 5:143-163. 
Ericsson, J. \& Reneby, J. (2001). The evaluation of corporate liabilities: Theory and tests. Mcgill University and Stockholm School of Economics. Working Paper.

Hull, J. C. \& White, A. (2000). Valuing credit default swaps. Journal of Derivatives, 8(1):29-40.

Israel, R. B., Rosenthal, J. S., \& Wei, J. Z. (2001). Finding generators for markov chains via empirical transition matrices, with applications to credit ratings. Mathematical Finance, 11:245-265.

Jarrow, R. A., Lando, D., \& Turnbull, S. M. (1997). A Markov model for the structure of credit risk spreads. Review of Financial Studies, 10:481-523.

Jarrow, R. A. \& Turnbull, S. M. (1995). Pricing derivatives on financial securities subject to credit risk. Journal of Finance, 50:53-85.

Jones, P. E., Scott, P. M., \& Rosenfeld, E. (1984). Contigent claims analysis of corporate capital structures: An empirical investigation. Journal of Finance, 39(39):611-27.

Lando, D. (1998). On cox processes and credit-risky securities. Review of Derivatives Research, 2:99-120.

Lardic, S. \& Rouzeau, E. (1999). Implementing merton's model on the French corporate bond market. Crédit Commercial de France, Direction de la Recherche et de l'Innovation. Working Paper.

Leland, H. (1994). Corporate debt value bond covenants and optimal capital structure. Journal of Finance, 49:1213-1252.

Merton, R. C. (1974). On the pricing of corporate debt: The risk structure of interest rates. Journal of Finance, 29:449-470.

Schonbucher, P. J. (2000). Credit risk modelling and credit derivatives. Tese de Doutorado, Universidade de Bonn.

Shimko, D., Tejima, N., \& Van Deventer, D. R. (1993). The pricing of risky debt when interest rates are stochastic. J. Fixed Income, 3(2):58-65.

Vasicek, O. (1984). Credit valuation. KMV Corporation. Working paper.

Zhou, C. (1996). A jump-diffusion approach to modelling credit risk and valuing defautable securities. Federal Reserve Board, Washington. Working Paper. 